\title{
Improvement of sagittal balance and lumbar lordosis following less invasive adult spinal deformity surgery with expandable cages and percutaneous instrumentation
}

\author{
Clinical article
}

\author{
Michael Y. WANG, M.D. \\ Department of Neurological Surgery, University of Miami Miller School of Medicine, Miami, Florida
}

\begin{abstract}
Object. The treatment of adult spinal deformity (ASD) remains a challenge for the spine surgeon. While minimally invasive surgery (MIS) has many favorable attributes that would be of great benefit for the ASD population, improvements in lordosis and sagittal balance have remained elusive in cases involving the MIS approach. This report describes the evolution of an MIS method for treating ASD with attention to sagittal correction.

Methods. Over an 18-month period 25 patients with thoracolumbar scoliosis were treated surgically. The mean patient age was 72 years, and $68 \%$ of the population was female. Patients were treated with multilevel facet osteotomies and interbody fusion in which expandable cages (mean 3.2 levels) were placed and percutaneous screw fixation (mean 5.3 levels) was performed. Seven patients underwent supplemental percutaneous iliac fixation.

Results. All patients underwent MIS without conversion to a traditional open procedure. The mean operative time was 273 mins and the mean blood loss was $416 \mathrm{ml}$. There were no intraoperative complications. The Cobb angle over the scoliotic deformity improved from a mean of $29.2^{\circ}$ to that of $9.0^{\circ}(\mathrm{p}<0.001)$. Lumbar lordosis between L-1 and S-1 improved from a mean of $27.8^{\circ}$ to one of $42.6^{\circ}(\mathrm{p}<0.001)$. Sagittal vertical axis improved from $7.4 \mathrm{~cm}$ to $4.3 \mathrm{~cm}(\mathrm{p}=0.001)$. Numeric pain scale scores improved as well, an average of 3.3 and 4.2 for the leg and back, respectively. A mean improvement of 20.8 points on the Oswestry Disability Index was seen at 12 months. Complications included: two cases requiring hardware repositioning, one case of screw pullout, one asymptomatic pedicle screw breach, prolonged hospitalization from constipation, and one acute coronary syndrome developing 3 days after surgery without myocardial damage.

Conclusions. An expanding body of evidence suggests that sagittal balance remains a keystone for good outcomes after ASD surgery. Minimally invasive surgery that involves a combination of osteotomies, interbody height restoration, and advanced fixation techniques may achieve this goal in patients with less severe deformities. While feasibility will have to be proven with larger series and improved surgical methods, the present technique holds promise as a means of reducing the significant morbidity associated with surgery in the ASD population.

(http://thejns.org/doi/abs/10.3171/2012.9.SPINE111081)
\end{abstract}

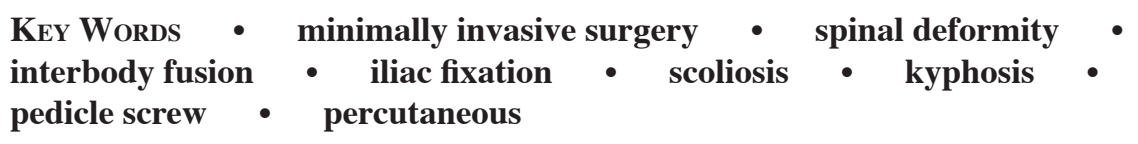

$\mathrm{S}$ URGERY for ASD remains a challenging proposition. A combination of factors acts in concert to create the milieu for high intraoperative and postoperative complication rates. These factors include more medical comorbidities, patient de-conditioning due to pain and immobility, associated osteoporosis, a rigid skeletal deformity, and abnormal spinal anatomy. ${ }^{7,13}$ In addition, the surgical intervention necessary to treat these patients is

Abbreviations used in this paper: $\mathrm{AP}=$ anteroposterior; $\mathrm{ASD}=$ adult spinal deformity; BMI = body mass index; MIS = minimally invasive surgery; NPS = numeric pain scale; ODI = Oswestry Disability Index; SVA = sagittal vertical axis; TLIF = transforaminal lumbar interbody fusion. typically a long-segment fusion with fixation and osteotomies, often in conjunction with interbody fusion or anterior access. The end result is a painful and debilitating operation that requires prolonged anesthesia, a long recovery period, and extended hospital stays. Thus, it is not surprising that reports from expert deformity surgeons reveal a high rate of serious complications. For example, in a series of 361 patients reported by Pateder and colleagues, ${ }^{14}$ the 30 -day mortality rate was $2.4 \%$. In 2011, Smith et al. ${ }^{18}$ analyzed data from the Spinal Deformity Study Group and reported that $26.2 \%$ of their 206 patients suffered a minor complication and $15.5 \%$ suffered a major complication.

Despite these statistics, the outcomes from ASD sur- 
gery can be highly rewarding for the patient. ${ }^{4,17}$ Recently, many surgeons have attempted to leverage the purported benefits of MIS to treat deformity problems. ${ }^{2,15,20}$ Indeed, claims of reduced blood loss, lower infection rates, and quicker mobilization would appear to address many of the major drawbacks of ASD surgery. ${ }^{16}$ Thus, a variety of techniques have evolved in an attempt to apply MIS methodologies to correct coronal and sagittal deformities. To date, most methods have focused on using a combined MIS lateral transpsoas interbody procedure and subsequent percutaneous screw placement. This technique, undertaken with much enthusiasm, has proven excellent for treating local degenerative arthritis, restoring foraminal height, achieving indirect neural decompression, and correcting coronal deformity. ${ }^{2,3,5,9,20}$

However, 3 significant drawbacks remain with the transpsoas interbody fusion/percutaneous screw placement approach: 1) the need for lateral and prone positioning prolongs anesthesia time in this medically compromised patient population; 2) the inability to treat the lumbosacral junction without an additional surgical approach; and 3) only minimal or modest improvements in lumbar lordosis. In a recently reported series of 35 patients by Acosta et al., ${ }^{1}$ the lateral MIS approach allowed for a Cobb angle correction from $21.4^{\circ}$ preoperatively to $9.7^{\circ}$ postoperatively, a statistically significant improvement. However, lumbar lordosis only changed from $42.1^{\circ}$ to $46.2^{\circ}$, respectively, despite improvements in interbody height. Overall, the global sagittal alignment was unchanged. This is similar to the $5^{\circ}$ improvement in global lordosis noted by Karikari et al. ${ }^{10}$ Given the importance of maintaining or improving sagittal parameters in the ASD population, this value represents an important deficit in this particular MIS technique for addressing ASD.

For the past decade, several MIS groups have attempted to close the gap between MIS and open surgery outcomes. Radiographically, the ideal MIS would achieve sagittal and coronal deformity correction to a degree similar to that of open procedures. I report here my experience using multilevel minimally invasive TLIF performed via a unilateral exposure in concert with the placement of percutaneous screws for treating ASD in an effort to meet these goals.

\section{Methods}

\section{Patient Population}

A consecutive series of 25 patients received treatment during an 18-month period at a single institution. All patients undergoing expandable interbody cage placement for ASD correction were included. Cobb angles demonstrated greater than $20^{\circ}$ of scoliosis in all patients. All deformities were rigid with less than $10^{\circ}$ of motion in the coronal or sagittal planes across the deformity segments on flexion, extension, and lateral bending radiographs. However, patients with a Cobb angle of more than $60^{\circ}$ were excluded from this surgical procedure. In all patients conservative measures had also failed, and all patients had severe back and/or back and leg pain with distance-limited gait. The minimum follow-up period af- ter surgery was 12 months. The study received University of Miami institutional review board exemption.

All patients underwent preoperative CT scanning and 36-in standing radiography to assess spinal alignment. Cobb angles were used to measure the degree of scoliosis. This was determined by measuring the maximal coronal angulation between the two most angulated upper vertebral endplates on 36-in standing radiographs. Global lumbar lordosis was determined by measuring the sagittal angulation between lines drawn parallel to the upper endplates of L-1 and S-1. The SVA was measured by dropping a plumb line from the anterior inferior aspect of the C-7 vertebra. The minimum distance from this line to the posterior superior endplate of S-1 was the SVA measured in centimeters. Coronal balance was measured on AP 36-in radiographs and was determined as the offset between a line drawn vertically between the pedicles of S-1 and T-1.

\section{Surgical Technique}

The surgery is performed after induction of general anesthesia and with the patient lying prone. Positioning on the Jackson table is critical to allow the belly to hang and increase lordosis after the releasing osteotomies and disc removal. A single midline incision is made over the segments to be treated. This technique prevents prolonged skin bleeding and cosmetic defects, problems often seen with multiple stab incisions. A plane is created above the superficial fascia so that percutaneous screws can be placed with minimal disruption of the soft-tissue envelope. Only one side of the spine is accessed to allow for facetectomies and interbody cage placement. A subperiosteal dissection is then taken to the lateral facet joints only on the side of approach for the minimally invasive TLIF, and the contralateral side is not exposed below the fascia. The choice of which side to approach from will depend on the type of deformity, clinical symptoms, and the goals of surgery. I typically approach from the concavity of the fractional curve (the curve at the lumbosacral junction), which is the same side as the convexity of the major curve (typically midlumbar).

Following exposure and confirmation of spinal levels, the facet osteotomies are performed from lateral to medial across the superior facet just rostral to the pedicle. A cerebellar retractor is used to elevate the soft-tissue envelope. A midline laminectomy is not typically performed unless there is severe central canal stenosis that requires direct decompression (Fig. 1). An osteotomy is performed at each minimally invasive TLIF level. The operating microscope is then used to more clearly visualize the critical structures. Bone, joint, and soft-tissue removal is then taken medially up to the lateral border of the ligamentum flavum as a minimum. The lateral anulus fibrosus is then found just rostral to the pedicle, and the surrounding veins are secured using bipolar cautery. An incision is made through the anulus and insert-and-rotate shaver dilators are used to remove the intervertebral disc, with great care taken to preserve the cortical vertebral endplates. This is particularly important in the setting of osteoporosis. In addition, the medial angulation of the approach is critical and will differ by level. In surgeries in 

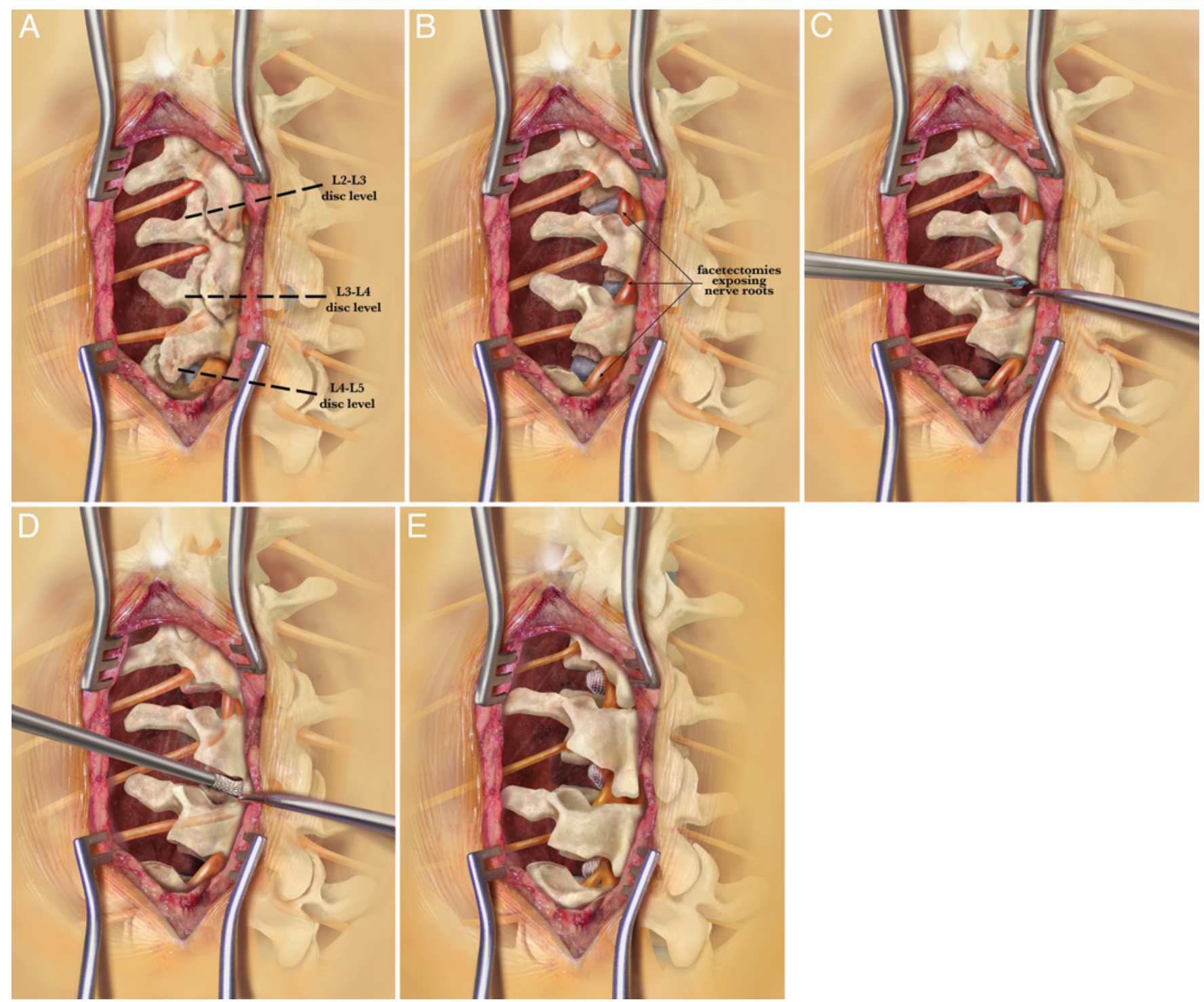

FIG. 1. Technique for interbody cage placement. A: Soft-tissue exposure of the facet joint complexes. B: Facetectomies expose the lateral disc anulus. C: Intervertebral disc removal in preparation for cage placement and fusion. D: Expandable cage placement through a small portal $7 \mathrm{~mm}$ in diameter. E: Cage expansion, which partially corrects the deformity.

which the approach is on the side of the concavity (simple curves without a fractional component), the disc removal will be predominantly ipsilateral to distract the interspace that has been closed down. In surgeries in which the approach is on the side of the convexity of the major curve (also the side of the concavity of the fractional curve), a steep approach is taken so that the contralateral disc is accessed and removed so that interspace height can also be restored on the collapsed portion of the major curve.

Once complete disc removal has been achieved, fusion adjuvants are placed into the disc space. I have used autograft bone from the facetectomies, as well as rhBMP-2 (InFuse, Medtronic Sofamor Danek) at a dose of up to $1.05 \mathrm{mg} / \mathrm{level}$. A 25-mm Optimesh cage (Spineology) is then inserted and internally filled with allograft granular matrix. The device is inserted through a 7-mmdiameter portal and inflated within the disc space, restoring intervertebral height. It should be noted that both of these products used in this setting are considered off label by the FDA. Once filled and inflated, the cages are crimped shut and the nerve roots inspected to ensure there is no impingement.

Percutaneous screws are then placed by cannulating the pedicles with Jamshidi needles. This is accomplished primarily using AP fluoroscopic radiographs (Fig. 2). The technique involves docking the needle tip at the junction of the transverse process and lateral facet joint. The needle is tapped $2 \mathrm{~cm}$ into the bone without passing the medial wall of the pedicle on AP radiographs. This use of the AP technique allows accurate compensation for axial rotation in complex ASD cases. Iliac screws are placed using the obturator outlet view for percutaneous cannulation as previously described. ${ }^{19}$ For iliac screws a window is made in the posterior superior iliac spine to avoid screw head prominence. Each needle is then exchanged for a $\mathrm{K}$ wire. An insulating sheath protects the soft tis- 

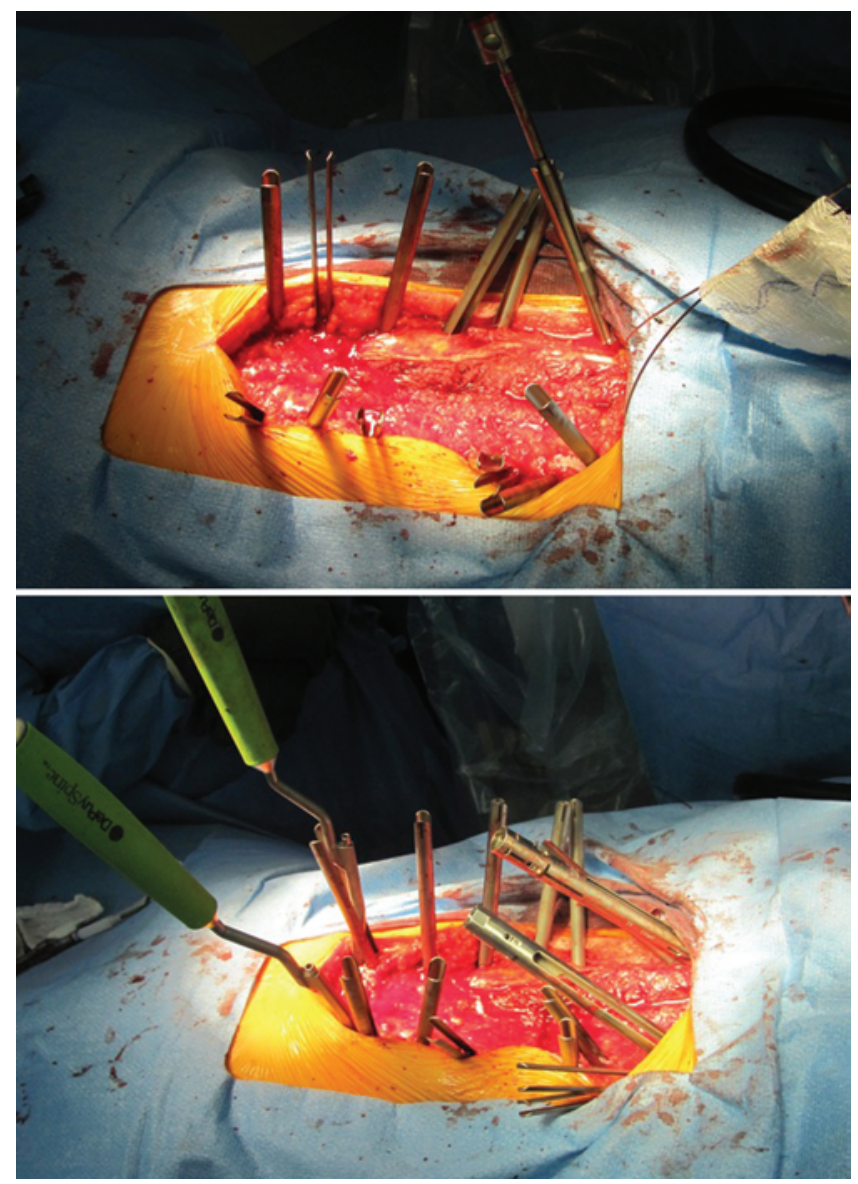

Fig. 2. Upper: Pedicle and iliac screws with extension tabs placed through the muscle and fascia from T-11 to the pelvis. Lower: Bilateral rod passed through the screw heads to correct the deformity and enhance lordosis.

sues, while an awl and tap create the path for subsequent pedicle screw (Viper, DePuy Spine) placement.

Rods are placed subfascially by passage through the screw extensions. At the levels not treated with an interbody cage, a posterolateral fusion was performed. This is accomplished by decorticating the lamina and facet joint through the same fascial incision used for screw placement. Autograft bone and remaining rh-BMP-2 were used to fuse these levels at the thoracolumbar junction. Rod de-rotation, compression of screw heads along the major curve convexity, and mating of the rod to the screw heads are used to complete the deformity correction. The surgical sites are then closed in standard fashion over suction drainage.

\section{Results}

A total of 25 patients (17 women and 8 men) were treated using this technique. The mean age was 72 years (range 62-84 years). The mean height and weight were $163.2 \mathrm{~cm}$ and $74.3 \mathrm{~kg}$, respectively, and the overall average BMI was 27.8 (range 21.3-43.5). The mean weighted Charlson comorbidity index score was 1.0 and the combined Charlson score mean was 3.8. A total of 132 segmental levels (mean 5.28/patient) and 80 interbody levels (3.2/patient) were treated. A total of 256 percutaneous pedicle screws and 14 percutaneous iliac screws were placed. The series was consecutive with no patients lost to follow-up.

All surgeries were successfully completed without conversion to an open procedure (Table 1). The mean operative time from skin incision to final closure was 273 mins (range 180-360 mins). The surgical blood loss, as measured by the perfusionist, averaged $415.6 \mathrm{ml}$ (range 200-800 ml). Ninety-two of patients were out of bed and ambulating on the 1st postoperative day; the mean stay in acute care was 5 days (range 3-8 days). Overall, $80 \%$ of patients were discharged to home and $20 \%$ were sent to inpatient rehabilitation.

\section{Radiographic Outcomes}

Radiographic outcomes were determined using preand postoperative 36-in standing radiographs acquired at the last follow-up visit (Figs. 3 and 4), which was at 12 months. The mean preoperative Cobb angle was $29.2^{\circ} \pm$ $9.3^{\circ}$ (range $20^{\circ}-59^{\circ}$ ). This improved to a mean of $9.0^{\circ} \pm$ $5.0^{\circ}$ (range $3^{\circ}-21^{\circ}$ ). This decrease reflected an average

TABLE 1: Summary of patient demographic data and radiographic results

\begin{tabular}{|c|c|c|}
\hline Parameter & Mean \pm SD & Range \\
\hline \multicolumn{3}{|l|}{ demographics } \\
\hline age (yrs) & $72 \pm 7$ & $62-84$ \\
\hline BMI & $27.8 \pm 5.0$ & $21.3-43.5$ \\
\hline combined Charlson score & $3.8 \pm 1.4$ & $2-7$ \\
\hline \multicolumn{3}{|l|}{ operative characteristics } \\
\hline no. of treated levels & $5.28 \pm 2.15$ & $3-9$ \\
\hline no. of interbody levels & $3.2 \pm 0.76$ & $1-4$ \\
\hline operative time (mins) & $273 \pm 50$ & $180-360$ \\
\hline intraop blood loss (ml) & $415.6 \pm 149$ & $200-800$ \\
\hline \multicolumn{3}{|l|}{ radiographic results } \\
\hline preop Cobb angle $\left({ }^{\circ}\right)$ & $29.2 \pm 9.3$ & $20-59$ \\
\hline postop Cobb angle $\left({ }^{\circ}\right)$ & $9.0 \pm 5.0$ & $3-21$ \\
\hline preop lumbar lordosis $\left({ }^{\circ}\right)$ & $27.8 \pm 12.9$ & $6-53$ \\
\hline postop lumbar lordosis $\left({ }^{\circ}\right)$ & $42.6 \pm 12.1$ & $19-66$ \\
\hline preop SVA (cm) & $7.4 \pm 4.9$ & $0-17.5$ \\
\hline postop SVA (cm) & $4.3 \pm 5.7$ & $0-13.0$ \\
\hline \multicolumn{3}{|l|}{ clinical outcomes } \\
\hline \multicolumn{3}{|l|}{ NPS score (extremity) } \\
\hline preop & $5.1 \pm 2.02$ & $2-8$ \\
\hline postop & $1.8 \pm 0.99$ & $0-4$ \\
\hline change & 3.3 & $0-7$ \\
\hline \multicolumn{3}{|l|}{ NPS score (axial back) } \\
\hline preop & $7.6 \pm 1.70$ & $4-10$ \\
\hline postop & $3.4 \pm 1.70$ & $0-8$ \\
\hline change & 4.2 & $2-8$ \\
\hline \multicolumn{3}{|l|}{ ODI score } \\
\hline preop & $44.9 \pm 11.8$ & $18-66$ \\
\hline postop & $24.1 \pm 11.6$ & $4-50$ \\
\hline change & 20.8 & $6-34$ \\
\hline
\end{tabular}


M. Y. Wang

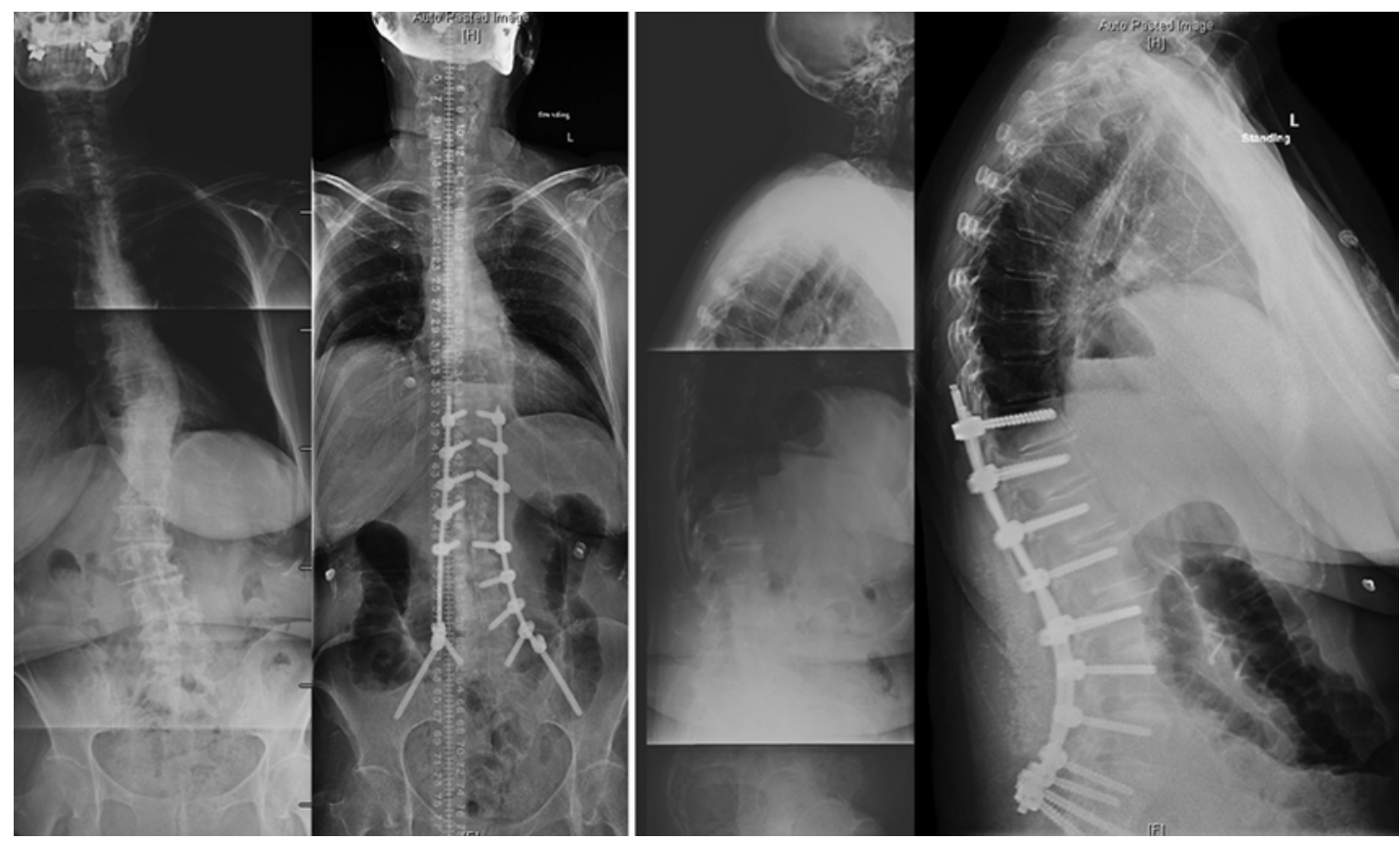

FIG. 3. Representative case showing a T-10 to iliac fusion: pre- and postoperative AP radiographs (left) and pre- and postoperative lateral 36 -in radiographs (right).

$20.2^{\circ}$ of improvement, which was statistically significant ( $\mathrm{p}<0.001)$. The mean preoperative global lumbar lordosis was $27.8^{\circ} \pm 12.9^{\circ}\left(\right.$ range $\left.6^{\circ}-53^{\circ}\right)$. This parameter improved to a mean of $42.6^{\circ} \pm 12.1^{\circ}$ (range $19^{\circ}-66^{\circ}$ ), reflecting an average of $14.78^{\circ}$ of improvement that was also statistically significant $(\mathrm{p}<0.001)$. The mean preoperative SVA was 7.4 $\pm 4.9 \mathrm{~cm}$ (range $0-17.5 \mathrm{~cm}$ ). This improved to a mean of 4.3 \pm 5.7 (range $0-13.0 \mathrm{~cm}$ ), reflecting an average improvement of $3.2 \mathrm{~cm}$ that was a statistically significant $(\mathrm{p}=0.001)$. Fourteen patients had at least $1 \mathrm{~cm}$ of coronal imbalance before surgery (range $0-5 \mathrm{~cm}$ ). Preoperatively the mean coronal imbalance was $1.64 \mathrm{~cm}$, whereas postoperatively it was $0.72 \mathrm{~cm}$, representing a mean improvement of 0.92 $\mathrm{cm}$. Three patients had worsening of coronal balance due to straightening of the major curve without addressing the fractional curve adequately. In 2 of these patients coronal balance worsened from $1 \mathrm{~cm}$ to $3 \mathrm{~cm}$, and in another patient it worsened from $2 \mathrm{~cm}$ to $3 \mathrm{~cm}$ (Fig. 5).

All patients underwent 12-month postoperative CT scanning. Fusion status was verified as complete at all levels in 22 of the 25 patients. All interbody fusion sites demonstrated evidence of bridging bone across the intervertebral disc space (Fig. 6). In all 3 cases in which fusion could not be verified, this occurred at the thoracolumbar
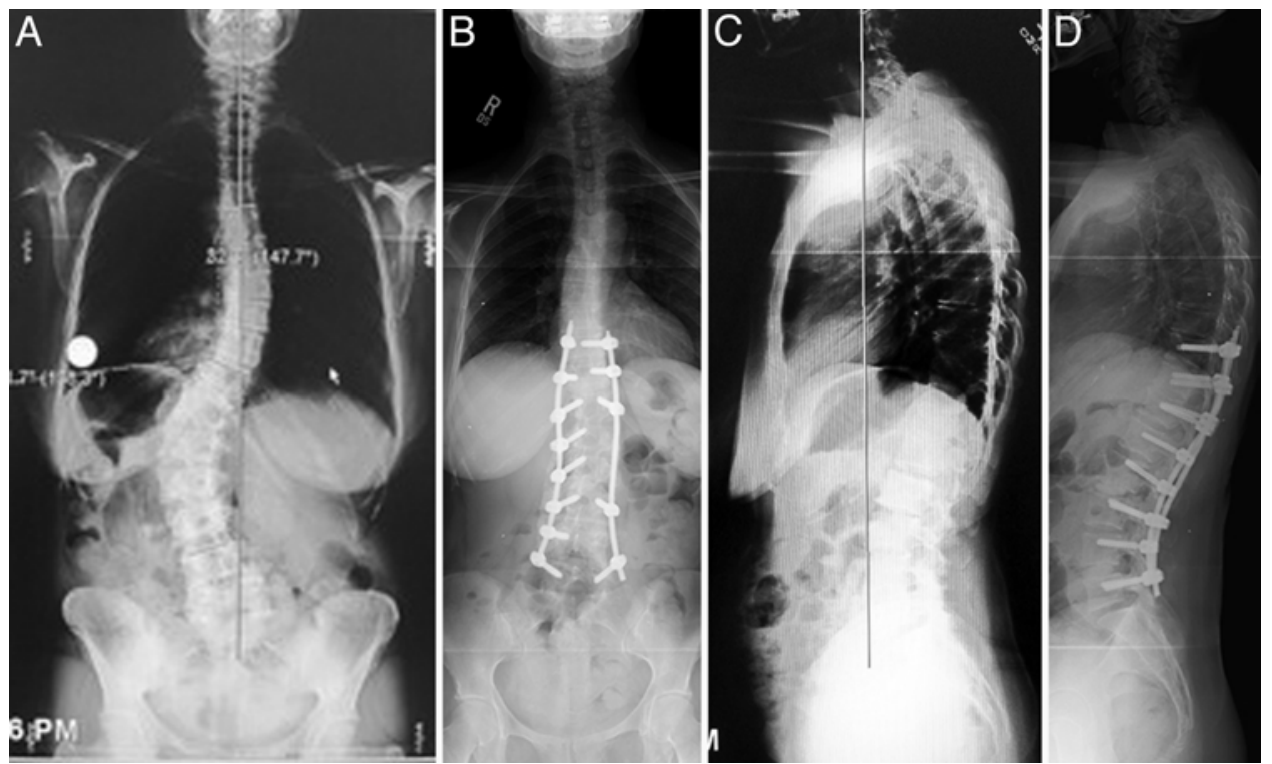

FIG. 4. Representative case demonstrating a T10-L5 fusion: pre- and postoperative AP radiographs ( $A$ and $B$ ) and pre- and postoperative lateral 36 -in radiographs ( $C$ and $\mathrm{D})$. 


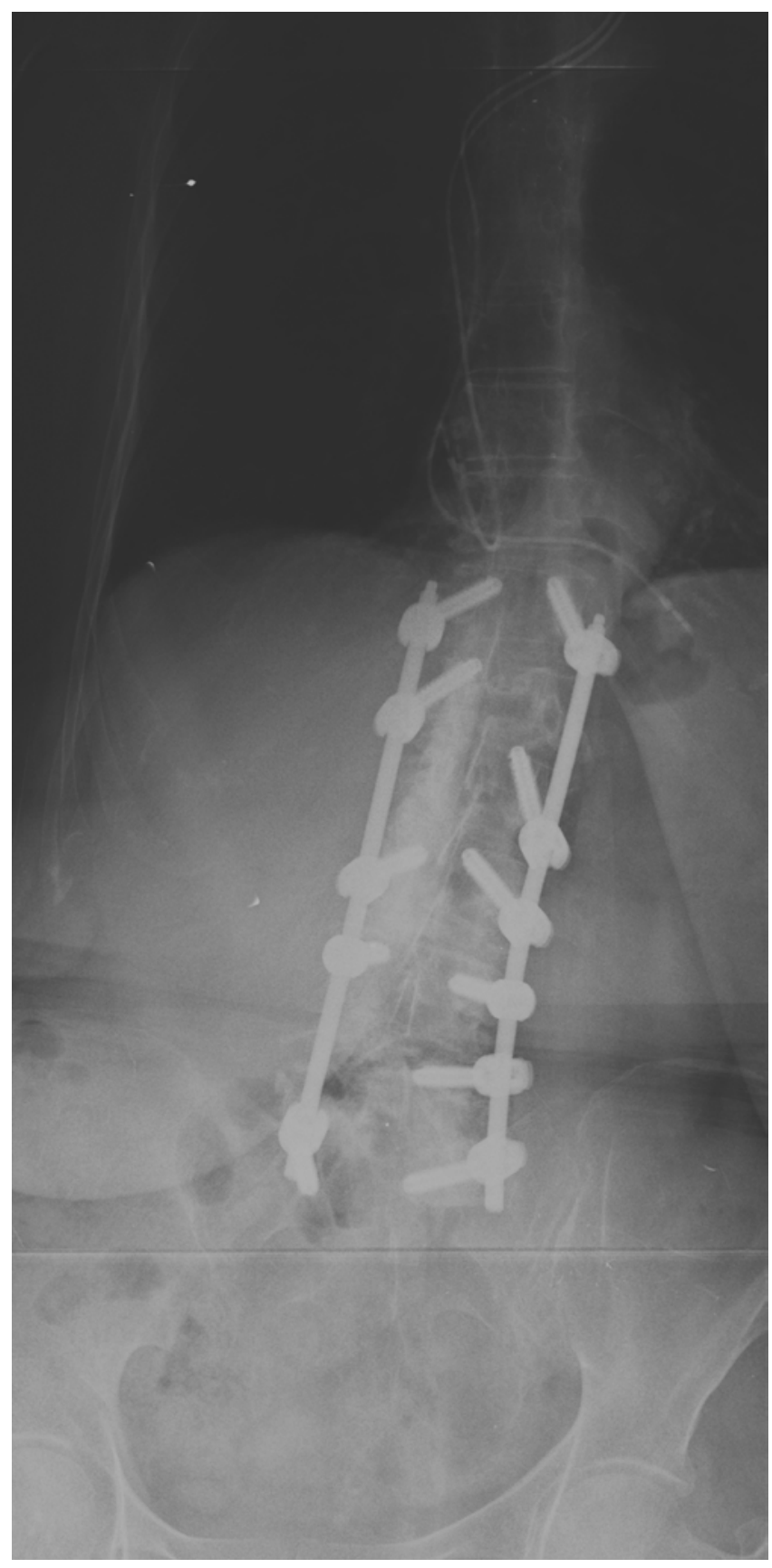

FIG. 5. Representative case showing 1 of the 3 patients with inadequate management of the fractional curve, resulting in suboptimal coronal alignment. Inadequate management may result in the need for future revision surgery.

junction where no interbody fusion was performed. This resulted in a total of $3(5.8 \%)$ of 52 such treated levels. In 1 case there was some evidence of screw loosening and in the other 2 levels there was a paucity of bony growth in the posterolateral or interlaminar space. However, these patients did not experience any local symptoms of pseudarthrosis as of the last follow-up.

\section{Clinical Outcomes}

Clinical outcomes were determined using the NPS for the lower extremities (leg and buttock symptoms)

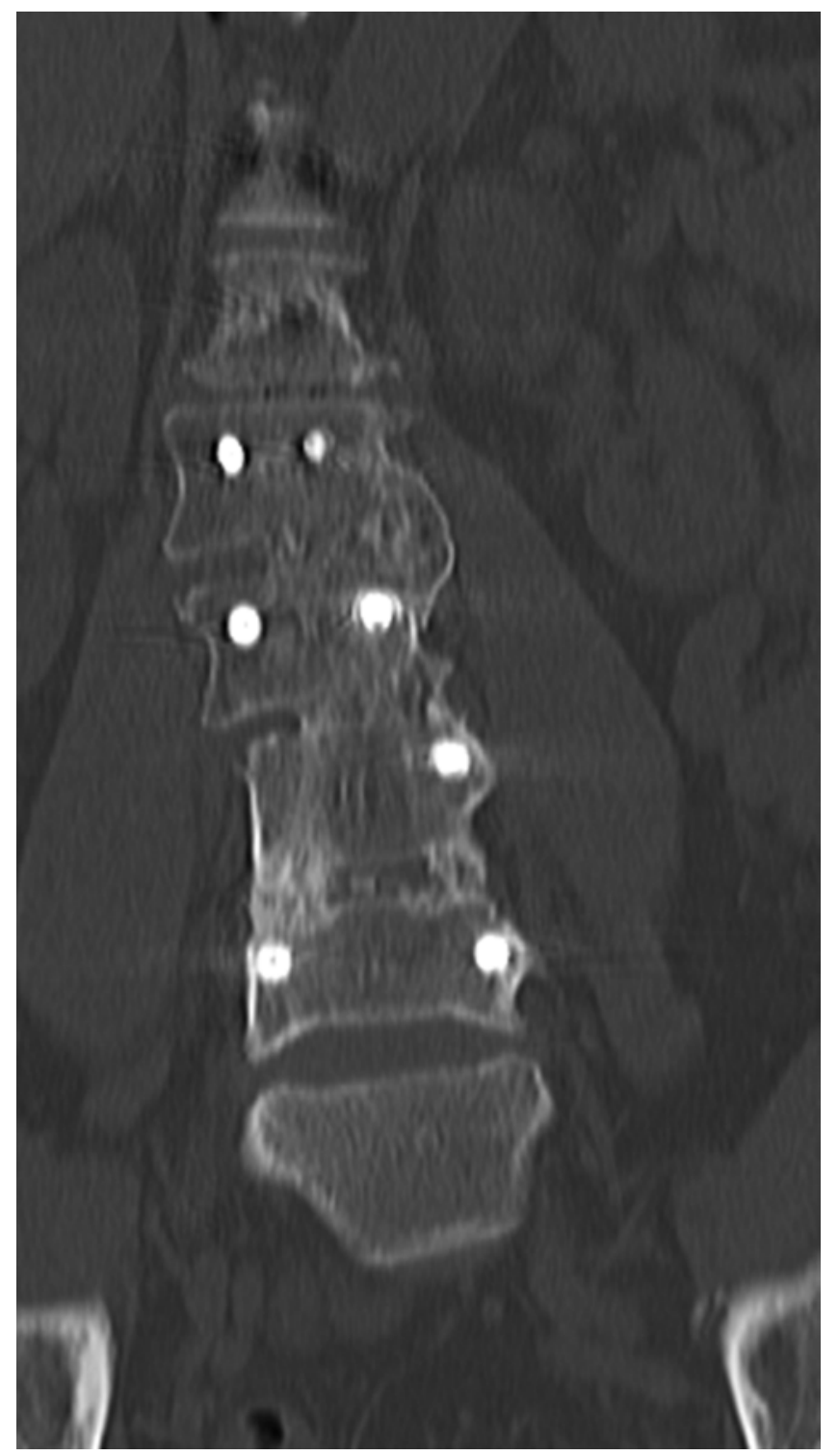

FIG. 6. Representative case of a patient who underwent T11-L4 fusion for kyphosis. The coronal CT scan shows solid interbody fusion at L1-2, L2-3, and L3-4.

and low back (axial symptoms); scores range from 1 to 10 , with 10 being the highest. The ODI was also used. Questionnaires were completed in the clinic setting before surgery and afterward at the 12-month follow-up. Preoperatively, the NPS for leg pain was 5.1, improving to 1.8 after surgery, reflecting a mean improvement of 3.3 (Table 1). Preoperatively, the NPS for axial back pain was 7.6, improving to 3.4 after surgery, reflecting a mean improvement of 4.2. Preoperatively, the ODI score was 44.9 , improving to 24.1 after surgery, reflecting a mean improvement of 20.8 .

\section{Complications}

There were no cases of neurological worsening except for one that involved new dorsiflexor weakness of the foot. Of the 16 patients with preoperative nerve root pain, all but one had resolution of leg pain. A total of 6 com- 
plications occurred. Four were hardware-related complications. Two patients had a medial L-5 screw breach. In one case the screw was removed 2 weeks after the index surgery due to foot weakness. In the other case the patient only had numbness and the screw was removed after confirmation of solid bony fusion 9 months after surgery. One patient had an asymptomatic Grade II screw breach at T-10. One patient had early screw pullout at S-1 and the screw was removed 1 year later after confirmation of successful L5-S1 fusion. Thus, a total of 3 patients returned to the operating room during the follow-up period, one of whom had an acute complication and two of whom had delayed complications. The fifth complication was a case of acute coronary syndrome in a 79-year-old woman who did not suffer permanent myocardial ischemia. The final complication occurred in a patient with a prolonged hospitalization course due to severe constipation. This patient was ultimately discharged to home.

\section{Discussion}

Minimally invasive surgery has tremendous potential as a treatment for medically and skeletally compromised ASD patients. In the past surgeons performing MIS have primarily focused on short-segment fusions for degenerative disease. ${ }^{16}$ Traditional deformity surgeons, recognizing the deficiencies of the minimally invasive approach, have more or less refrained from these methods to treat their patients. This has been largely due to the limited ability of MIS techniques to increase global lumbar lordosis. In this report I describe my initial experience with a combination of less invasive techniques for treating ASD with the intention of meeting the same goals as a traditional open surgery deformity corection. This proposition has been difficult in ASD because of the stiffness of the spine and ubiquitous osteoporosis, which biomechanically compromises the bone-screw interface.

A previous clinical series by Scheufler and coworkers $^{15}$ demonstrated the efficacy of multilevel minimally invasive TLIF to obtain improved lordosis in the ASD population. In that series of 30 patients, image guidance was used to plan and place hardware for interbody and transpedicular fixation. An excellent mean correction of $31.7^{\circ}$ and $44.8^{\circ}$ in the coronal and sagittal planes, respectively, was achieved. Thus, the use of TLIF for deformity surgery, as described by Heary and Karimi, ${ }^{8}$ appears to afford the opportunity for improved deformity correction.

Using a somewhat different method of unilateral access with facet osteotomies, multilevel minimally invasive TLIF, and percutaneous instrumentation, we were able to achieve a modest but significant improvement in sagittal alignment and a good improvement in coronal alignment. Furthermore, the surgery is accomplished with the patient in a single position, thus reducing anesthesia time. In the present series the total operative time was comparable to that of open surgery but with significantly reduced blood loss. While the skin incision is long and a subperiosteal dissection is used on a portion of the spine, opening the soft-tissue envelope on only one side has real benefits for the patient. First, the limited muscular exposure minimizes blood loss and reduces the operative time for opening and wound closure. Second, because half of the paravertebral musculature remains intact, patients are mobilized rapidly and are typically ambulatory less than 24 hours after surgery. This has substantially reduced the postoperative problems of deep venous thrombosis, atelectasis, and pneumonia that can be related to prolonged immobilization frequently seen following ASD surgeries. Drazin et al. ${ }^{6}$ found the incidence of pulmonary complications to be as high as $64 \%$. Perhaps because of the reduced hemodynamic stress and rapid ambulatory status, pulmonary problems were not encountered in this case series despite a mean patient age of 72 years.

\section{Long-Segment Constructs and Iliac Fixation}

Because of the complexity and heterogeneity of the present patient population, many key surgical points have become apparent when treating ASD with an minimally invasive surgical approach. Many of the tools and techniques of ASD surgery have heretofore been unavailable for MIS application. For example, rod cross-linking has been a challenge. Nevertheless, major advances in longsegment and iliac MIS instrumentation are becoming increasingly feasible for the treatment of a multitude of spinal pathologies. In treating ASD, the passage of a double-curved rod across both the thoracolumbar and lumbosacral junctions can be difficult. This is particularly true with a very lordotic lumbar curvature. In some cases, this has even limited the amount of lordotic correction that could be achieved. Rod passage is certainly made easier with some curvature in the rod, but acute bends can make it very difficult to pass without damaging the soft tissues. The bend in the tip of the rod can be used to "steer" it through the screw heads during cranial to caudal passage.

Iliac fixation allows for a long-segment construct to be anchored securely in osteoporotic patients. ${ }^{12}$ Long iliac screws also allow the caudal hardware construct to be anchored at a point anterior to the pivot point, helping to maintain sagittal alignment. However, connection to the hardware construct can be challenging even in open surgery cases. In almost all cases an offset connector is unnecessary with proper planning. Starting the iliac screw more inferiorly and the S-1 screw more superiorly allows for greater rod length between these two adjacent screw heads. A slight lateral rod bend helps to facilitate the more dorsal and lateral location of the iliac screw saddle.

\section{Importance of the Fractional Curve}

One of the major mistakes in the early portion of this series had been a failure to adequately respect and address the fractional curve (Fig. 5). In 3 cases a satisfactory straightening of the midlumbar and major curve resulted in coronal imbalance because the base at the lumbosacral junction was angulated. With increased experience, this issue, which has long been well understood by traditional deformity surgeons, has been addressed by accessing the spine from the concavity of the fractional curve. Because interbody spacers at the lumbosacral junction will typically occupy the disc space more on the side of access, this side of an approach will tend to elevate and correct the fractional curve. 


\section{Approach From the Major Curve's Convexity}

Accessing the spine from the major curve's convexity allows the surgeon to perform several other key steps successfully. First, because the spine will be rotated toward the side of the approach, the interbody cage can more easily be placed into the contralateral side of the spine. In the midlumbar spine, this allows the cages to be preferentially applied across the midline onto the concavity of the major curve. Because axial rotation can be as great as $35^{\circ}$, the need for a steep angle of approach through the soft tissues is reduced. Second, because the osteotomies are performed on the convexity, compression of the screw heads will result in both scoliosis correction and an improvement in lumbar lordosis.

\section{Use of Expandable Cages}

Applying expandable cages represents a key advance in minimally invasive deformity surgery. Using a mesh bag that can be later filled with demineralized bone matrix, the intervertebral space can be accessed through the Kambin triangle. This device is placed through a 7-mm portal and thus requires little or no neural retraction and can be inflated to a final maximal diameter of $25 \mathrm{~mm}$. However, it should be noted that this application represents an off-label use of the product as specified by the FDA. Other devices are also available for accomplishing this task by entering through a small portal to ultimately expand to a larger intervertebral height.

\section{Study Deficiencies}

This series represents my second foray into treating complex ASD with an attempt to minimize the factors that contribute to the high morbidity rate of the surgery. I had initially used a lateral approach as the primary mechanism for achieving interbody height and coronal curve correction. However, sagittal correction was only possible with a second position surgery (percutaneous pedicle screws), requiring either one prolonged anesthetic session or a staged approach with a long hospital stay. However, the radiographic results in this series are inferior to open surgery, particularly compared with 3-column osteotomies..$^{11,13}$ Greater improvement in the SVA will be necessary for the treatment of severe coronal imbalance in patients with fixed deformities. It should also be noted that severe scoliotic curves $\left(>60^{\circ}\right)$ were not treated with these methods and are still currently managed using an open procedure at my institution. Long-term results in a large, multiinstitutional cohort will also be necessary to demonstrate the maintenance of corrections and the generalizability of these results.

This study is also limited by the relatively short follow-up time period. While radiographic evidence of successful fusion was demonstrated, ASD patients may develop instrumentation failures many years after surgery. Thus, imaging that shows bridging bone may not guarantee against a delayed instrumentation failure in some patients. Of particular importance is the proximal end of the construct where there is less bone surface area for a posterolateral fusion compared with that of traditional open surgery. Thus, the bulk of the high-quality fusion materials are used at the two most cranial levels. Ultimately long-term follow-up of 5 or more years will be required for definitive technique validation.

\section{Future Directions}

Significant gaps remain in the MIS spectrum of techniques for treating complex spinal problems in challenging patient populations. Future advances in the ability to mobilize the spine prior to realignment, decrease rates of pseudarthrosis, safely place implants for fixation, and initiate powerful corrective maneuvers will be necessary to advance the field if a minimally invasive approach is to achieve the results seen with powerful 3-column osteotomies. However, improvements in spinal instrumentation, image guidance, and ostobiologics will likely make minimally invasive ASD surgery a viable option for the increasingly aging population.

\section{Conclusions}

An expanding body of evidence suggests that sagittal balance remains a keystone for good outcomes after ASD surgery. Minimally invasive surgery that involves a combination of osteotomies, interbody height restoration, and advanced fixation techniques may achieve this goal in patients with less severe deformities. While feasibility will have to be proven with larger series and improved surgical methods, the present technique holds promise as a means of reducing the significant morbidity associated with surgery in the ASD population.

\section{Disclosure}

Dr. Wang is a consultant for DePuy Spine and Aesculap Spine and receives royalty payments from DePuy Spine, Inc.

\section{References}

1. Acosta FL, Liu J, Slimack N, Moller D, Fessler R, Koski T: Changes in coronal and sagittal plane alignment following minimally invasive direct lateral interbody fusion for the treatment of degenerative lumbar disease in adults: a radiographic study. Clinical article. J Neurosurg Spine 15:92-96, 2011

2. Anand N, Baron EM, Thaiyananthan G, Khalsa K, Goldstein TB: Minimally invasive multilevel percutaneous correction and fusion for adult lumbar degenerative scoliosis: a technique and feasibility study. J Spinal Disord Tech 21:459-467, 2008

3. Anand N, Rosemann R, Khalsa B, Baron EM: Mid-term to long-term clinical and functional outcomes of minimally invasive correction and fusion for adults with scoliosis. Neurosurg Focus 28(3):E6, 2010

4. Bess S, Boachie-Adjei O, Burton D, Cunningham M, Shaffrey C, Shelokov A, et al: Pain and disability determine treatment modality for older patients with adult scoliosis, while deformity guides treatment for younger patients. Spine (Phila Pa 1976) 34:2186-2190, 2009

5. Dakwar E, Cardona RF, Smith DA, Uribe JS: Early outcomes and safety of the minimally invasive, lateral retroperitoneal transpsoas approach for adult degenerative scoliosis. Neurosurg Focus 28(3):E8, 2010

6. Drazin D, Shirzadi A, Rosner J, Eboli P, Safee M, Baron EM, et al: Complications and outcomes after spinal deformity surgery in the elderly: review of the existing literature and future directions. Neurosurg Focus 31(4):E3, 2011

7. Fehlings MG, Ibrahim GM: Editorial. Spinal deformity. J Neurosurg Spine 13:663-665, 2010 
8. Heary RF, Karimi RJ: Correction of lumbar coronal plane deformity using unilateral cage placement. Neurosurg Focus 28(3):E10, 2010

9. Isaacs RE, Hyde J, Goodrich JA, Rodgers WB, Phillips FM: A prospective, nonrandomized, multicenter evaluation of extreme lateral interbody fusion for the treatment of adult degenerative scoliosis: perioperative outcomes and complications. Spine (Phila Pa 1976) 35 (26 Suppl):S322-S330, 2010

10. Karikari IO, Nimjee SM, Hardin CA, Hughes BD, Hodges TR, Mehta AI, et al: Extreme lateral interbody fusion approach for isolated thoracic and thoracolumbar spine diseases: initial clinical experience and early outcomes. J Spinal Disord Tech 24:368-375, 2011

11. Kim YJ, Bridwell KH, Lenke LG, Cheh G, Baldus C: Results of lumbar pedicle subtraction osteotomies for fixed sagittal imbalance: a minimum 5-year follow-up study. Spine (Phila Pa 1976) 32:2189-2197, 2007

12. Lippman CR, Salehi SA, Liu JC, Ondra SL: Salvage technique of posterior iliac bolt placement in long-segment spinal constructs with a previous posterior iliac crest harvest: technical note. Neurosurgery 58 (1 Suppl):ONS-E178, 2006

13. Mummaneni PV, Dhall SS, Ondra SL, Mummaneni VP, Berven S: Pedicle subtraction osteotomy. Neurosurgery 63 (3 Suppl):S171-S176, 2008

14. Pateder DB, Gonzales RA, Kebaish KM, Cohen DB, Chang JY, Kostuik JP: Short-term mortality and its association with independent risk factors in adult spinal deformity surgery. Spine (Phila Pa 1976) 33:1224-1228, 2008

15. Scheufler KM, Cyron D, Dohmen H, Eckardt A: Less invasive surgical correction of adult degenerative scoliosis, part
I: technique and radiographic results. Neurosurgery 67:696710,2010

16. Schwender JD, Holly LT, Rouben DP, Foley KT: Minimally invasive transforaminal lumbar interbody fusion (TLIF): technical feasibility and initial results. J Spinal Disord Tech 18 (Suppl):S1-S6, 2005

17. Smith JS, Shaffrey CI, Berven S, Glassman S, Hamill C, Horton $\mathrm{W}$, et al: Improvement of back pain with operative and nonoperative treatment in adults with scoliosis. Neurosurgery 65:86-94, 2009

18. Smith JS, Shaffrey CI, Glassman SD, Berven SH, Schwab FJ, Hamill CL, et al: Risk-benefit assessment of surgery for adult scoliosis: an analysis based on patient age. Spine (Phila Pa 1976) 36:817-824, 2011

19. Wang MY, Ludwig SC, Anderson DG, Mummaneni PV: Percutaneous iliac screw placement: description of a new minimally invasive technique. Neurosurg Focus 25(2):E17, 2008

20. Wang MY, Mummaneni PV: Minimally invasive surgery for thoracolumbar spinal deformity: initial clinical experience with clinical and radiographic outcomes. Neurosurg Focus 28(3):E9, 2010

Manuscript submitted December 13, 2011.

Accepted September 24, 2012.

Please include this information when citing this paper: published online October 26, 2012; DOI: 10.3171/2012.9.SPINE111081.

Address correspondence to: Michael Y. Wang, M.D., Lois Pope LIFE Center, Department of Neurological Surgery, 1095 NW 14th Terrace, Miami, Florida 33136. email: MWang2@med.miami.edu. 